\title{
Current Advances and Challenges in HIV-1 Vaccines
}

\author{
Isaac R. Rodriguez-Chavez, PhD, Mary Allen, RN, MS, Edgar \\ L. Hill, MSPH, Rebecca L. Sheets, PhD, Michael Pensiero, PhD, \\ James A. Bradac, PhD, and M. Patricia D'Souza, PhD
}

\author{
Corresponding author \\ M. Patricia D'Souza, PhD \\ Division of AIDS, National Institute of Allergy and Infectious \\ Diseases, National Institutes of Health, 6700-B Rockledge Drive- \\ MSC 7628, Room 5130, Bethesda, MD 20892, USA. \\ E-mail: pdsouza@niaid.nih.gov \\ Current HIVIAIDS Reports 2006, 3:39-47 \\ Current Science Inc. ISSN 1548-3568 \\ Copyright () 2006 by Current Science Inc.
}

Recent advances in science, which have aided HIV-I vaccine development, include an improved understanding of HIV-I envelope structure and function, expansion of the pipeline with innovative vaccine strategies, promising multi-gene and multi-clade vaccines that elicit cellular immunity, conduct of clinical trials in a global network, and development of validated techniques that enable simultaneous measurement of multiple $T$ cell vaccineinduced immune responses in humans. A common feature of several preventive vaccine strategies now in early clinical trials is their ability in nonhuman primates to attenuate clinical disease rather than completely prevent HIV-I infection. One vaccine concept has been tested in largescale clinical trials, two are currently in efficacy trials, and one more is poised to enter efficacy trial in the next few years. Simultaneously, expanded efforts continue to identify new designs that induce mucosal immunity as well as broadly neutralizing antibodies.

\section{Introduction}

HIV-1 is the etiologic agent of AIDS. More than $40 \mathrm{mil}-$ lion people worldwide are infected, about 14,000 new infections occur daily, and millions of deaths occur yearly. The epidemic is growing exponentially, and over $90 \%$ of infected people live in developing countries and are unable to afford or barely have access to existing antiretroviral therapies (ART). Despite the implementation of national and international initiatives to provide ART in many developing countries, toxicities, long-term expenses, and imminent ART-driven viral resistance emphasize the urgent need for a preventive vaccine. A safe, effective, preventive, and accessible vaccine is the best public health strategy to prevent disease.

The scientific, financial, and logistic challenges to accomplishing this objective are formidable. Scientifically, HIV-1 is genetically diverse and the relevance of the different genetic clades to vaccine design remains a subject of active debate. Whether any animal model predicts vaccine efficacy in humans remains unknown, and there are no validated laboratory correlates of immune protection. Despite these obstacles, globally, more than 100 vaccine candidates have been tested since 1987, including more than 80 products and 14 adjuvants in clinical trials. This review describes vaccine strategies currently under investigation in preclinical and clinical trials and summarizes the current assays used to assess vaccine-induced immune responses.

\section{Role of Vaccine-induced Humoral Immunity}

Vaccine-induced broadly cross-reactive neutralizing antibodies (NAbs) can prevent virus entry if present in sufficient quantities at the time of infection. The induction of NAbs has been challenging due to the multiple mechanisms that the virus (envelope [Env] changes) utilizes to evade immune responses. First, the high degree of Env glycosylation and an evolving glycan shield mask key binding sites involved in virus attachment and fusion $[1,2]$. Second, conserved neutralizing epitopes located in the gp120-CD4 binding region are thermostably hidden [3]. Third, the antigenic variation of gp120 creates a conformational hindrance for the immune system by concealing the recognition of epitopes surrounding receptor binding sites [4]. Fourth, the complex HIV-1 life cycle, coupled with the high mutation rate of the reverse transcriptase induces escape mutants that evade cellular and humoral immunity [5].

Env (gp160) plays a critical role in virus binding, fusion, entry, infection, and pathogenesis. Therefore, NAbs directed at Env can prevent HIV-1 entry. Broad and potent NAbs are rarely observed either in patients with HIV-1 infection or in Env-immunized individuals. The isolation of only a small number of human cross-neutral- 
izing monoclonal antibodies (mAbs) (IgG b12, 2G12, 447-52D, 4E10, and 2F5) testifies to the rarity of this type of immunity [6]. The first three mAbs are gp120-specific, whereas the latter two are gp41-specific. Although the role of NAbs in virus-infected patients has been debated, there is increasing evidence of their ability to prevent infection. Passive immunization with NAbs at sufficiently high titers can protect against virus challenge. A recent study to assess the protective potential of a cocktail of three human neutralizing mAbs (2G12, 2F5, and 4E10) in infected subjects that underwent ART interruption showed that two of eight chronically infected individuals had delayed viral rebound. In contrast, four of six mAb treated acutely infected subjects had a substantial delay in viral rebound upon ART interruption [7••]. Furthermore, escape mutant analysis demonstrated that the activity of 2G12 was central for the in vivo neutralizing effect observed in the triple antibody (Ab) cocktail. This evidence supports the principle that NAbs restrain HIV1 replication in infected subjects, but that virus escape occurs rapidly.

Recently, it has also been demonstrated that 2F5 and $4 \mathrm{E} 10$ are polyspecific autoantibodies directed against

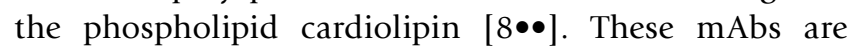
characterized by having long hydrophobic complementary-determining regions (CDR3) typically found in naturally occurring polyspecific autoantibodies of indi-

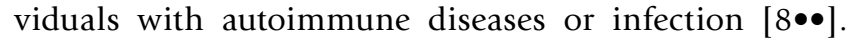
Autoreactive B lymphocytes carrying long CDR3 segments are routinely removed from B-cell pools or become tolerant to autoantigens. Thus, the presence of conserved, neutralizing epitopes in HIV-1 that mimic autoantibody epitopes may represent a viral evolutionary strategy to escape membrane proximal-directed humoral immunity, a mechanism described as antigenic mimicry.

\section{Assays Used to Assess Vaccine-induced Humoral Immunity}

Accurate measurement of vaccine immunogenicity depends on utilizing validated assays in laboratories that comply with guidelines for good clinical laboratory practices. Together these processes assure reliable, repeatable, and auditable results to give assurance to sponsor and regulatory authorities about assay proficiency at multiple laboratories. It also permits data comparability across trials and networks worldwide so that only the most efficacious vaccines move forward.

Current humoral immune assays in use are the enzyme-linked immunosorbent assay [9] and Western Blot [10] to measure Ab binding capacity and virus neutralization to measure Ab functional ability [11•]. Assays must be high-throughput, correlative, and validated. In recent years, new technologies utilizing stable cell lines expressing CD4 and HIV-1 coreceptors have improved assessment of vaccine-elicited NAbs. Also, a reference panel for clade B HIV-1 viruses comprising of Env clones from recently transmitted viruses has been created [11•]. Previously, assessments of the neutralizing Ab response involved the use of multiple HIV-1 strains. A lack of uniformity in the choice of strains used by different investigators has made it difficult to interpret and compare existing data. Thus, the availability and standard use of this and other nonclade $B$ reference reagent panels should allow consistent comparisons of the potencies and breadths of elicited NAbs across trials and laboratories. In turn, this will facilitate the prioritization of candidate vaccines in preclinical and clinical studies.

\section{Novel Env Approaches}

Two trials to assess the efficacy of monomeric, recombinant gp120 vaccine were recently completed in 2003 [12]. Results from these trials showed that these vaccines were unable to elicit NAbs against neutralization-sensitive domains of HIV-1 and did not prevent infection $[12,13]$. A great deal of effort has been devoted to the construction of native, oligomeric Env immunogens that elicit broad and potent cross-reactive NAbs against neutralization-sensitive domains of HIV-1. These approaches include the use of: 1) glycosylation site-mutated Env glycoproteins; 2) variable loop-deleted Env glycoproteins; 3) CD4-Env glycoprotein complexes, including the use of single chain chimeric gp120 complexes that express codon optimized gp120 sequences and CD4 domains linked in a single peptide to form intrachain complexes [14]; and 4) modified CD4-independent Env glycoproteins $[15,16]$. The first two approaches described are based on the exposure of conserved neutralizing epitopes on Env upon removal of its masking elements. Immunogenicity studies indicate that such modified Env products are unable to increase the breadth and potency of induced NAbs [17,18]. The latter two approaches described are based on the use of modified Env glycoproteins that mimic one of the fusion intermediates generated during viral entry. These approaches induce broadly cross-reactive NAbs as well as strong anti-CD4 responses (third approach) or require further immunogenicity evaluation (fourth approach).

Based on the existing evidence, the design of novel immunogens capable of eliciting NAbs will at least require further understanding of the oligomeric and complex structure of Env [19]; the use of innovative Env modifications; the use of specific immunization strategies to potentially induce novel autoreactive antibodies with neutralizing properties similar to 2F5 and $4 \mathrm{E} 10$; the potential combination of several Env glycoproteins from multiple clades to expand the breadth of NAbs [20]; a further understanding of the role that 2F5, 4E10, and other NAbs may play in preventative vaccines and the production and characterization of new human NAbs. 
Role of Vaccine-induced Cellular Immunity Most vaccines currently in the development pipeline are designed to stimulate strong cell-mediated immune responses to HIV-1. This rationale is based on published observations that CD8 + cytotoxic T lymphocytes (CTLs) appear to play an important role in controlling HIV-1 and simian immunodeficiency virus (SIV) replication and in clearing the infection, as evidenced by 1) their presence in infected macaques decreases viral replication and reduces disease progression; 2) high numbers of specific CTLs can be detected in long-term nonprogressors (LTNP) $[21,22]$ and in persistently seronegative subjects who have been vastly exposed to HIV-1 but remain uninfected [23]; 3) viremia declines in acute infection is coincident with the expansion of HIV-1 specific CTLs; and 4) escape mutants are generated under CTL's pressure. Although CTL responses are promptly detected in most infections, their magnitude is usually insufficient to control viral load and disease progression. Consequently, an effective vaccine will most likely require CTL induction as part of protective immunity along with triggering other immune responses.

\section{Assays to Assess Vaccine-induced Cellular Immunity}

CTLs are capable of multiple effector functions including cytolysis and production of various cytokines and chemokines. In HIV-1 vaccine trials, CTL function is currently measured with assays such as interferon gamma (IFN- $\gamma$ ), ELISpot, and intracellular staining by flow cytometry (ICS). Although ELISpot is robust, sensitive and inexpensive for rapid screening of antigen-specific CTLs and epitope mapping, it does not provide information regarding the immuno-phenotype of responder lymphocytes and only measures production of a single effector molecule, IFN- $\gamma$. In contrast, ICS correlates better with CTL responses and in the multi-parameter format can provide more information simultaneously concerning the specific T-lymphocyte subset, its maturational phenotype and multiple functional profiles. Recent data have reported that IFN- $\gamma$ is not consistently associated with control of HIV-1 replication [24] and that examination of IFN- $\gamma$ secretion alone will underestimate the total of anti-HIV-1 specific CD4+ T lymphocytes, because many secrete interleukin (IL)-2 alone. Furthermore, it was recently shown in LTNP that the $\beta$-chemokine macrophage inflammatory protein (MIP)-1 $\beta$ dominates CTL responses and that the most frequent profile observed was the co-expression of IFN- $\gamma$, MIP-1 $\beta$, and CD107a [24].

Other potential assays of use in vaccine end-point measurements include the tetramer assay that directly tests the frequency of CD8+ T lymphocytes with a specific receptor and, if combined with multicolor flow cytometry, allows phenotype identification. However, the drawback is that reagent preparation requires knowledge of the subject's
HLA type. Another assay that measures cell proliferation is also gaining use because recent data suggest that it is the proliferative capacity, rather than the quantity of CTLs that is most relevant to controlling HIV-1 replication. The proliferative capacity upon antigen exposure is one functional attribute of CTLs that inversely correlates with viral load. CD8+ T lymphocyte proliferation appears to be coupled to perforin expression, which contributes to the killing ability of infected cells. Several studies done in individuals with HIV-1 infection have shown disruptions in T lymphocyte maturation processes, suggesting proliferation as the critical linking event. Future immunogencity tools are likely to include microarrays to identify specific gene profiles (clusters) that might be associated with developing protective immune responses against HIV-1 upon vaccination. The ability to probe and measure large numbers of genes simultaneously offers a distinctive advantage over current established methodologies (ELISpot, ICS) that measure a limited number of molecules.

\section{Preclinical and Clinical Vaccine Strategies}

Preliminary data from preclinical trials in animal models indicate that vaccine candidates do not prevent infection, but rather modify the course of disease progression. Consequently, the endpoints of future efficacy trials will need to monitor the ability of the vaccine to prevent infection as well as its effect on viral RNA levels and CD4+ T lymphocyte counts.

Current vaccine strategies include recombinant proteins expressed either by DNA plasmids or by viral vectors, and prime-boost strategies. The discussion below highlights those strategies furthest in clinical development (Fig. 1) along with some novel strategies in advanced preclinical development (Table 1) which are anticipated to move into clinical trials.

\section{DNA plasmids}

Some DNA vaccines express a single HIV-1 antigen whereas others express multiple antigens. Increasingly, candidates are based on the various circulating HIV-1 subtypes and most are codon-optimized to maximize expression of HIV-1 genes in human cells. In nonhuman primates, plasmid DNA has generally been safe when injected intramuscularly by needle or by a needleless injection device (Biojector ${ }^{\circledR}$, Bioject Medical Technologies, Inc., Portland, OR) or when adhered to gold beads and delivered epidermally using a gene gun. To enhance immune responses to DNA vaccines, it may be necessary to use high doses, administer separate plasmids for each vaccine antigen, and/or incorporate cytokines and other potential adjuvants into the vaccine formulation [25].

The National Institute of Allergy and Infectious Diseases (NIAID) Vaccine Research Center (VRC) is evaluating two DNA vaccines. The first vaccine comprises four plasmids: three modified envelope proteins from clades A, B, and C 


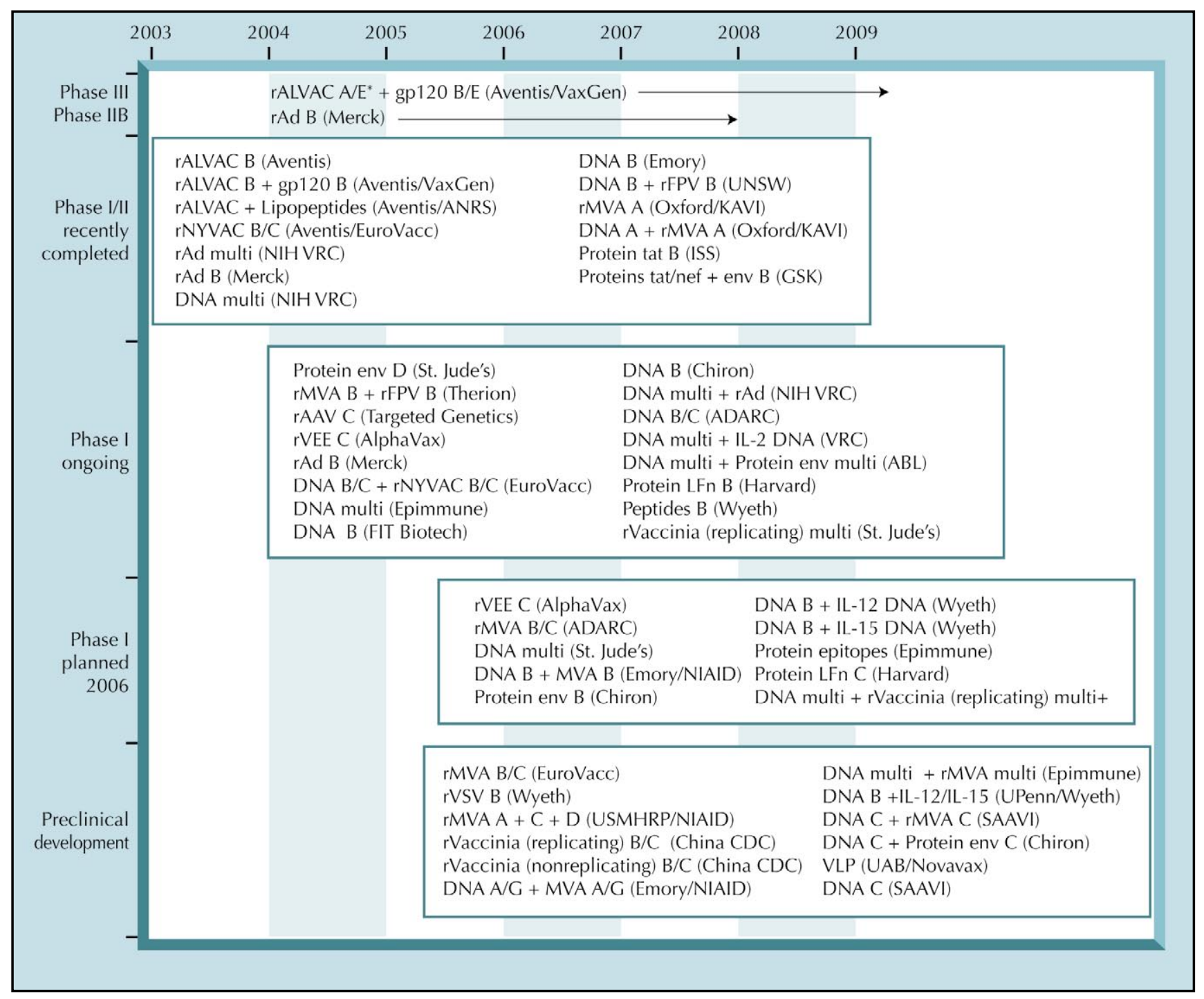

Figure 1. HIV-1 vaccines: clinical and preclinical pipeline. "A/E" (asterisk) refers to the HIV subtype on which the vaccine is based, in this case circulating recombinant form (CRF) A/E. Other subtypes in the figure include A, B, C, D, and CRFs B/C and A/G. "Multi" signifies that the vaccine contains HIV-1 genes, proteins, or peptides derived from multiple HIV-1 subtypes. AAV—adeno-associated virus; ADARC — Aaron Diamond AIDS Research Center; ANRS—Agence Nationale de Reseraches sur le Sida; CDC—Centers for Disease Control and Prevention; FPV—fowlpox virus; GSK—Glaxo SmithKline; ISS—Istituto Superiore di Sanità; KAVI—Kenyan AIDS Vaccine Initiative; MVA_Modified Vaccinia Ankara; NIAID—National Institute of Allergy and Infectious Disease; NIH VRC—National Institutes of Health Vaccine Research Center; SAAVI_South Africa AIDS Vaccine Initiative; UAB_-University of Alabama at Birmingham; UNSW_University of North South Wales; UPenn—University of Pennsylvania; USMHRP—US Military HIV Research Program.

and a Gag-Pol-Nef fusion protein from clade B; whereas the second VRC vaccine comprises six plasmids: the same A, B, and C env plasmids and also individual clade B gag, pol, and nef plasmids. Dose-ranging studies of as high as 8 $\mathrm{mg}$ of DNA found that $4 \mathrm{mg}$ and $8 \mathrm{mg}$ doses of the fourplasmid vaccine demonstrated equivalent immunogenicity, hence the lower dose is now being used in clinical trials. To date, both DNA vaccines are well tolerated and appear safe. Of note is that the four-plasmid DNA vaccine has induced immune responses to Env, but only weakly, if at all to the fusion protein antigens, Gag, Pol, and Nef. The six-plasmid DNA vaccine has generated broader immune responses to the multiple antigens in this product.
One approach to increase the potency of DNA is to include cytokines with the vaccine. In this regard, animals immunized with SIV gag and HIV-1 env DNA vaccines adjuvanted with IL-2/IgG fusion protein (to increase the half-life of the cytokine) or IL-2/IgG encoding plasmid maintained their CD4+ T lymphocyte numbers, exhibited low to undetectable viral loads and were protected from disease [26]. The four-plasmid DNA vaccine from the VRC described above is also being evaluated in combination with an IL-2/IgG DNA plasmid in an ongoing clinical trial.

Other DNA vaccines are being developed with additional cytokine gene adjuvants, such as IL-15 or IL-12. 


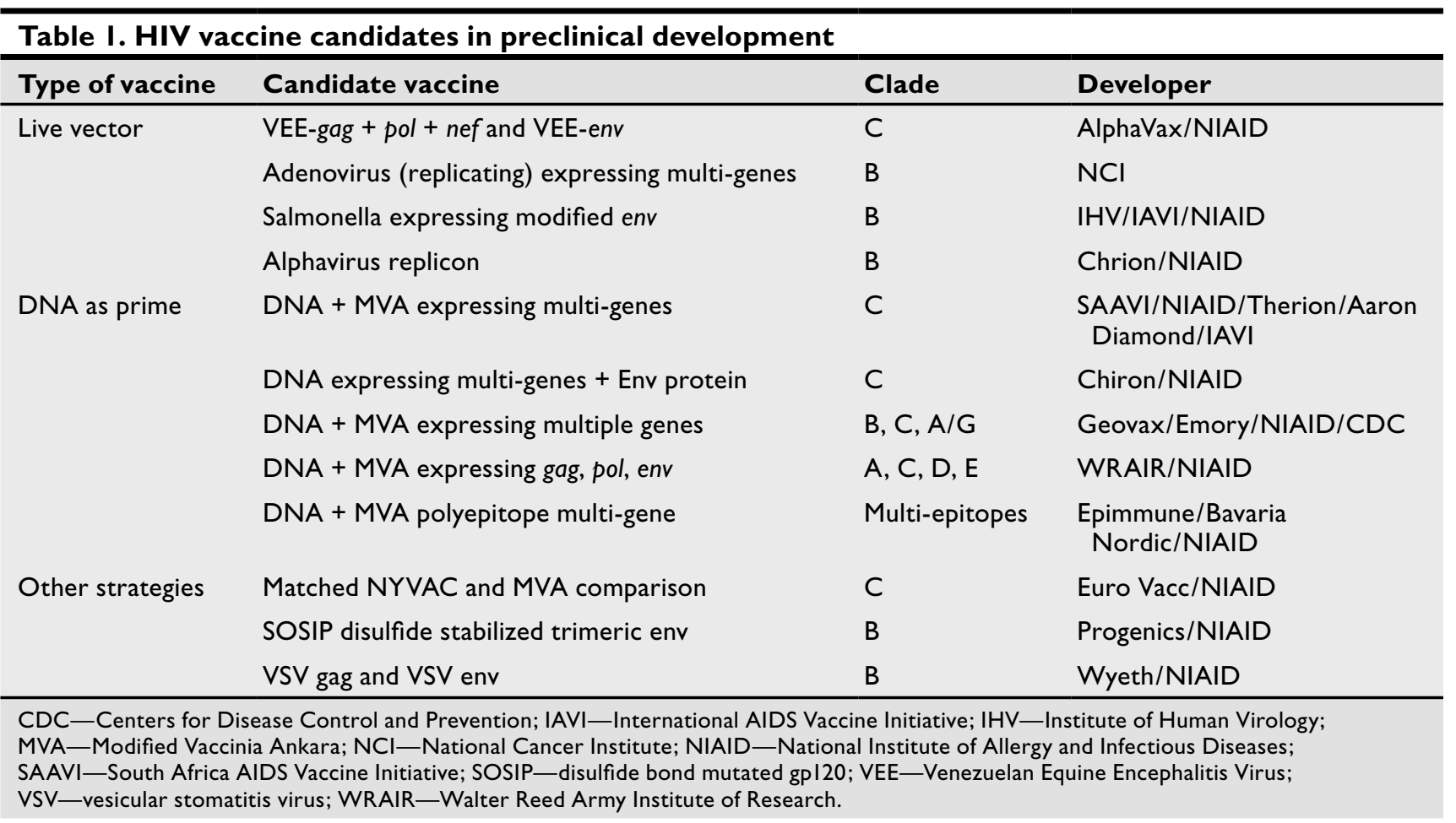

These vaccines are complex and some include a mixture of various HIV-1 genes or peptides representing CTL epitopes and the cytokine-expressing plasmid. Immunization of macaques with a gag-expressing DNA plasmid and a macaque IL-15 expressing plasmid resulted in an increase in magnitude of ELISpot responses. Similar results were observed with an IL-12-expressing plasmid adjuvant [27]. A recent clinical trial tested a peptide vaccine based on T helper epitopes from Env and Gag and CTL epitopes from Gag and Nef. The vaccine is formulated with a novel adjuvant (RC529-SE) and given with or without granulocyte macrophage colony stimulating factor (GM-CSF). A clinical trial using this as a boost to the DNA plasmids adjuvanted with IL-12 or IL-15 expressing plasmid adjuvants began in August 2005.

\section{Recombinant viral vectors}

After safety considerations, the ideal vector for HIV-1 vaccines should induce robust $\mathrm{T}$ - and B-lymphocyte immunity and provide persistent antigen stimulation for maintaining long lasting CD4 + and CD8+ T-lymphocyte responses. There is also a need for vectors and/or heterologous prime boosts that are not limited by preexisting immunity and immunogens that boost primes for long-lasting B-lymphocyte induction. Present evidence suggests that vector persistence leads to strong and durable immune response. The search for more persistent antigen presentation has focused on early persistent vectors including $\gamma$-2 herpesvirus, herpes virus replicons, measles virus, rabies virus and adeno-associated virus. Other vectors that offer high levels of expressed antigens are also being investigated including parainfluenza virus,
Enterovirus (poliovirus, Coxsackie, and parechovirus), Sendai virus, vesicular stomatitis virus, Listeria monocytogenes, Chlamydia trachomatis, Toxoplasma gondii, and attenuated Mycobacterium.

To date the most extensively studied viral vectors in human HIV-1 vaccine trials belong to the poxvirus family, including attenuated vaccinia vectors such as Modified Vaccinia Ankara (MVA) and NYVAC as well as Avipox viral vectors such as canarypox and fowlpox. MVA was used as a vector encoding SIV gag and pol to immunize macaques and was able to elicit antigen-specific CTL responses. Upon SIV challenge, vaccinated animals responded with a vigorous anamnestic CTL response and displayed reduced viremia that inversely correlated with the magnitude of the vaccine-induced CTL response [28]. These findings prompted development of recombinant MVA candidates for phase I trials. Bavarian Nordic has tested an MVA-nef vaccine given subcutaneously in individuals with history of smallpox vaccination in childhood, as well as vaccinia naïve individuals. MVA-nef was shown to be safe and immunogenic and induced a strong MVA-specific T lymphocyte and Ab response; however, few subjects showed responses to nef. Two poxvirus vectors, MVA and fowlpox, have been produced by Therion to contain either env/gag or tat/rev/nef inserts. These four vaccines are currently being tested in a combination approach. A highly attenuated form of vaccinia virus, NYVAC, expressing env, gag, pol, and nef from a clade C isolate of HIV-1 was recently evaluated in a clinical trial in the United Kingdom and Switzerland by EuroVacc. The vaccine was well tolerated, and in several subjects, vaccine-induced CTL responses were observed. 
Merck Research Laboratories (MRL) compared different replication-defective adenovirus vectored candidates encoding the SIVmac239 gag gene, administered to macaques and subsequently challenged with SHIV89.6P. Animals immunized with the adenovirus type 5 (Ad5) SIV gag vaccine prior to SHIV 89.6 challenge had the highest pre-challenge levels of cellular immunity as measured by ELISpot and tetramer assays, significantly reduced peak viral RNA, no loss of CD4 cells, and were protected from disease [29]. A MRL-developed vaccine is a co-formulation of three individual replicationdefective Ad5 vectors expressing HIV-1 clade B gag, pol, or nef and is being evaluated in the United States and internationally in a Phase IIb (proof-of-concept pilot efficacy) study. In earlier studies, subjects with low preexisting immunity to Ad5 (wild-type adenoviruses are a common cause of human disease) or who received the highest doses of vaccine were more likely to experience reactogenicity, although with subsequent doses, symptoms did not increase and the incidence of fever decreased. All dose levels elicited ELISpot responses although responses were lower in individuals with high titers $(>200)$ of pre-existing NAbs to Ad5. Cross-clade immunogenicity was also exhibited. MRL is exploring the vaccine potential of alternative types of adenoviruses (such as Ad24) that may be less affected by the problem of pre-existing antibodies than Ad5.

The VRC is also evaluating a multi-gene adenovirus vectored vaccine, at similar doses to those used in the MRL trial. The vaccine comprises separate adenovirus vectors, expressing a clade $\mathrm{B}$ Gag-Pol fusion protein; and Env from clades A, B, and C (see prime-boost below for further discussion). Researchers at NIAID and the National Cancer Institute (NCI) are developing a replication-competent Adenovirus (rAd4) recombinant vaccine that will prime cellular and humoral immunity and target mucosal inductive sites. In rAd4 immunized monkeys challenged with the highly pathogenic SIVmac251 isolate, control of viremia at the set point of infection was correlated with IFN- $\gamma$-secreting lymphocytes in response to Env and Rev peptides and reduced viremia during acute infection was correlated with Envspecific binding antibodies [30].

The Targeted Genetics adeno-associated virus vector expressing clade C HIV-1 gag, pol, and reverse transcriptase has expanded phase I trials from Belgium to Germany and to India to a recent phase II trial in South Africa. The vaccine is designed to elicit both humoral and cell-mediated responses. In nonhuman primates that received the recombinant adeno-associated virus-based vaccine, robust and durable $A b$ and T-lymphocyte responses were elicited and animals also had reduced viral load when challenged with a virulent strain of SIV [31].

Venezuelan Equine Encephalitis (VEE) is an alphavirus that synthesizes high levels of recombinant proteins and targets professional antigen presenting cells. A phase I trial testing AlphaVax's prototype attenuated VEE replicon vaccine expressing the gag gene is ongoing. Additionally, two VEE replicons expressing subtype C env or gag-nef-pol to be given simultaneously are expected to enter clinical trials in the coming year.

An attenuated vesicular stomatitis virus (rVSV) expressing the HIV-1 env and SIV gag genes was effective at preventing AIDS in a SHIV macaque model. Seven vaccinated monkeys were challenged with pathogenic SHIV89.6P and have remained healthy up to 14 months after challenge with low or undetectable viral loads [32]. In subsequent experiments rVSV was given either intramuscularly or intranasally to determine the optimal route of vaccine administration. Both routes resulted in significantly higher antigenspecific cellular immune responses as determined by class I tetramer staining, IFN- $\gamma$ ELISpot, and CTL assays than controls [33].

\section{Prime boost strategies}

To combine the strengths of various vaccine concepts to elicit either cellular or humoral responses and overcome their individual limitations, investigators are priming the immune system with one vaccine candidate and boosting with a heterologous product. Early clinical trials demonstrated that a viral vector prime and protein boost induced both cellular and humoral responses. Thus, a phase III, community-based trial of a canarypox recombinant vaccine (ALVAC-HIV-1 vCP1521) followed by a protein boost (AIDSVAX [B/E]) in 16,000 persons is being conducted in Thailand in a collaboration of the Thai government, the Walter Reed Army Institute for Research, and NIAID. ALVAC 1521 encodes gp120 from a clade E HIV-1 and portions of the gp41, gag, and protease genes from subtype $B$. This is the first vaccine efficacy trial of a prime-boost strategy.

Research at the VRC in collaboration with three international vaccine trial networks has resulted in initiation of three separate but harmonized trials of a prime with six-DNA plasmid mix expressing subtype B gag, pol, nef, and subtype $\mathrm{A}, \mathrm{B}$, and $\mathrm{C} e n v$ and boosted with a four-rAd5 mixture containing subtype B gag, pol, and subtype A, B, and $\mathrm{C} e n v$. The trials will be conducted in the Americas and southern and eastern Africa and are a prelude to an efficacy trial if the data support further clinical development. Preclinical data in macaques to support this strategy showed that the DNA plasmid prime followed by adeno-vector boost was capable of eliciting NAbs [34]. Further, the multi-clade prime-boost resulted in potent and broad cellular and humoral responses without antigenic interference [35].

Recombinant poxvirus vaccines have proven successful in boosting DNA-primed CTL responses to 10 to 100 times higher than those elicited by DNA or recombinant poxvirus immunizations alone in macaques. 
Priming with DNA may focus the immune response on the inserted genes, which then efficiently expands when poxvirus-infected cells express large amounts of protein and pro-inflammatory cytokines [36]. A clinical trial of this strategy should be initiated shortly. Another strategy is to test a series of matched inserts (clade $\mathrm{C} g a g$, pol, nef, and $e n v$ ) as a DNA prime with a poxvirus boost (either NYVAC or MVA from EuroVacc) or boost with both poxvirus and protein.

Aaron Diamond AIDS Research Center has developed an MVA vaccine and a two-plasmid DNA vaccine encoding env and gag, and pol, nef, and tat. Both vaccines are based on a clade $C$ strain of HIV-1 from a Chinese isolate. A DNA and MVA vaccine developed at Johns Hopkins University in the United States and produced in China entered clinical trials in China in Spring 2005. Clinical trials are near completion in the United Kingdom, Switzerland, Kenya, Uganda, and South Africa for clade A DNA and MVA vaccines that encode HIV-1 gag and a polypeptide of 25 CTL epitopes from Env, Gag, Pol, and Nef. The vaccines were well tolerated, but the immunogenicity did not meet milestones required for advanced clinical development.

\section{Immunobiology of HIV-1 Mucosal Infection}

Induction of mucosal immunity is central as HIV-1 enters CD4 + T lymphocytes residing in the mucosa-associated lymphoid tissue (MALT) of oral, rectal, and vaginal cavities. After entry, the virus also infects dendritic cells (DCs) and causes early memory CD4+ T-lymphocyte loss in the gut within days of infection and eventually in the body $[37,38,39 \bullet \bullet, 40 \bullet \bullet]$. Infected DCs spread the virus to adjacent resting or newly activated naïve CD4+ lymphocytes, causing local virus production at low levels. Once activated by antigens, T cells rapidly migrate and return home to effector lymphoid tissues. There, they become memory cells that coexpress high levels of CCR5 and CD4 and are permissive to HIV infection in effector lymphoid tissue. Thus, it is clear that most HIV replication occurs in mucosal tissues, particularly in acute infection so preserving or restoring mucosal $\mathrm{CD} 4+\mathrm{T}$ cells will be key to vaccine development.

HIV-1 specific CTL and Ab responses have been found in MALT upon viral challenge [41]. CTL responses have been detected in the cervical mucosa of HIV-1-exposed seronegative commercial sex workers [42] and in infected individuals. Furthermore, anti-HIV-1-specific CTL clones have been found circulating between MALT and blood [43]. Evidence has shown that secretory IgA has the ability to neutralize and to block transcytosis of HIV-1 crossing the mucosal epithelial cell layers by intracellular neutralization [44].

Local Ab and CTL responses in MALT upon initial infection may be an important complement to systemic immunity by reducing viral load at the por- tal of entry. Studies in macaques with SIV infection have shown that the turnover rate of memory CD4+ $\mathrm{T}$ lymphocytes in MALT dictates the rate of disease progression [45]. Similarly, studies in HIV-infected patients on ART have shown that viral replication does not stop in MALT [46]. Thus, early initiation of ART is critical to reconstitute the gut-associated $\mathrm{CD} 4+\mathrm{T}$ lymphocyte pools $[47,48]$.

Mucosal immunization of macaques with an HIV$1 /$ SIV peptide vaccine has been more effective against viral challenge than a systemic vaccine because vaccine-induced immune responses have been able to clear virus more effectively from the gut [49]. Some human vaccine strategies are able to generate and maintain mucosal immunity and memory at the genital mucosa [50]. Therefore, it is important to test candidate vaccines for their ability to induce mucosal responses, or for their ability to protect and preserve mucosal effector and memory anti-HIV-1 CD4+ T lymphocytes. However, further work is required to develop systematic assays for clinical assessment of mucosal immunity as well as are methods to predict efficacy of candidate mucosal vaccines.

\section{Conclusions}

The development of a successful HIV-1 vaccine is one of the toughest biomedical challenges facing society. The road to success will require coordination, information sharing, and collaboration from the global scientific community. Substantial progress made in HIV-1 vaccine design has been based on a broader understanding of the Env structure and its application to rationale vaccine design. There is a healthy preclinical pipeline of innovative vaccine concepts. Combinations of vaccine strategies that include multi-gene, multi-clade components with an ability to generate more potent CTL responses are currently in human trials. Trials are increasingly being conducted in regions of the world hardest hit by the HIV-1 epidemic with candidates appropriate to those regions. Moreover, the availability of validated immunogenicity assays has promoted comparison and prioritization of vaccine candidates globally for efficacy trials. An HIV-1 vaccine that is able to induce sterilizing immunity will need to embrace systemic as well as mucosal responses to block virus entry; and it is only by conducting well planned and ethical efficacy trials that we will develop the knowledge base and benchmarks to acquire and compare second-generation vaccine products.

\section{Acknowledgment}

The authors would like to thank Ms. Joanna Katzman for helpful discussions during the preparation of this manuscript. 


\section{References and Recommended Reading}

Papers of particular interest, published recently, have been highlighted as:

- Of importance

-• Of major importance

1. Kwong PD, Wyatt R, Robinson J, et al.: Structure of an HIV gp120 envelope glycoprotein in complex with the CD4 receptor and a neutralizing human antibody. Nature 1998, 393:648-659.

2. Wei X, Decker JM, Wang S, et al.: Antibody neutralization and escape by HIV-1. Nature 2003, 422:307-312.

3. Myszka DG, Sweet RW, Hensley P, et al.: Energetics of the HIV gp120-CD4 binding reaction. Proc Natl Acad Sci U S A 2000, 97:9026-9031.

4. Kwong PD, Doyle ML, Casper DJ, et al.: HIV-1 evades antibody-mediated neutralization through conformational masking of receptor-binding sites. Nature 2002, 420:678-682.

5. Preston BD: Reverse transcriptase fidelity and HIV-1 variation. Science 1997, 275:228-229.

6. Binley JM, Wrin T, Korber B, et al.: Comprehensive crossclade neutralization analysis of a panel of anti-human inmmunodeficiency virus type 1 monoclonal antibodies. J Virol 2004, 78:13232-13252.

7.•• Trkola A, Kuster H, Rusert P, et al.: Delay of HIV-1 rebound after cessation of antiretroviral therapy through passive transfer of human neutralizing antibodies. Nat Med 2005, 11:615-622.

This paper shows that a triple combination of neutralizing antibodies have the capacity to contain viremia in established human HIV-1 infection, but they must be potent and present at high doses to be effective. However, immune pressure by NAbs is subject to rapid escape, suggesting that a successful immunogen will probably have to elicit potent responses to multiple epitopes to limit viral evolution.

8.• Haynes BF, Fleming J, St Clair EW, et al.: Cardiolipin polyspecific autoreactivity in two broadly neutralizing HIV-1 antibodies. Science 2005, 308:1906-1908.

This paper demonstrates that the two most broadly reactive HIV-1 envelope gp41 human mAbs, 2F5 and 4E10, are polyspecific autoantibodies reactive with the phospholipid cardiolipin. This may explain why some key epitopes of the virus are poorly immunogenic.

9. Rawal BD, Degula A, Lebedeva L, et al.: Development of a new less-sensitive enzyme immunoassay for detection of early HIV-1 infection. J Acquir Immune Defic Syndr 2003, 33:349-355.

10. Sarngadharan MG, Popovic M, Bruch L, et al.: Antibodies reactive with human $T$-lymphotropic retroviruses (HTLV-III) in the serum of patients with AIDS. Science 1984, 224:506-508.

11. Li M, Gao F, Mascola JR, et al.: A reference panel of human immunodeficiency virus type 1 env clones from acute and early subtype $B$ infections for standardized assessments of vaccine-elicited neutralizing antibodies. J Virol 2005, 79:10108-10125.

This paper describes a panel of well-characterized reference strains of HIV-1 to be used for standardized assessments of vaccine-elicited NAbs. The use of standardized reagents and clonal viruses should allow the measurement of incremental improvements in breadth and potency of neutralization that might not otherwise be appreciated.

12. Flynn NM, Forthal DN, Harro CD, et al.: Placebo-controlled phase 3 trial of a recombinant glycoprotein 120 vaccine to prevent HIV-1 infection. J Infect Dis 2005, 191:654-665.

13. Gilbert PB, Peterson ML, Follmann D, et al.: Correlation between immunologic responses to a recombinant glycoprotein 120 vaccine and incidence of HIV-1 infection in a phase 3 HIV-1 preventive vaccine trial. J Infect Dis 2005, 191:666-677.
14. Devico A, Silver A, Thronton AM, et al.: Covalently crosslinked complexes of human immunodeficiency virus type 1 (HIV-1) gp120 and CD4 receptor elicit a neutralizing immune response that includes antibodies selective for primary virus isolates. Virology 1996, 218:258-263.

15. Hoxie JA, LaBranche CC, Endres MJ, et al.: CD4-independent utilization of the CXCR4 chemokine receptor by HIV-1 and HIV-2. J Reprod Immunol 1998, 41:197-211.

16. Kolchinsky P, Mirzabekov T, Farzan M, et al.: Adaptation of a CCR5-using, primary human immunodeficiency virus type 1 isolate for CD4-independent replication. J Virol 1999, 73:8120-8126.

17. Bolmstedt A, Sjolander S, Hansen JE, et al.: Influence of $\mathrm{N}$-linked glycans in $\mathrm{V} 4-\mathrm{V} 5$ region of human immunodeficiency virus type 1 glycoprotein gp160 on induction of a virus-neutralizing humoral response. J Acquir Immune Defic Syndr Hum Retrovirol 1996, 12:213-220.

18. Kim YB, Han DP, Cao C, Cho MW: Immunogenicity and ability of variable loop-deleted human immunodeficiency virus type 1 envelope glycoproteins to elicit neutralizing antibodies. Virology 2003, 305:124-137.

19. Wyatt R, Sodroski J: The HIV-1 envelope glycoproteins: fusogens, antigens, and immunogens. Science 1998, 280:1884-1888.

20. Chakrabarti BK, Ling X, Yang ZY, et al.: Expanded breadth of virus neutralization after immunization with a multiclade envelope HIV vaccine candidate. Vaccine 2005, 23:3434-3445.

21. Betts MR, Krowka JF, Kepler TB, et al.: Human immunodeficiency virus type 1-specific cytotoxic T lymphocyte activity is inversely correlated with HIV type 1 viral load in HIV type 1-infected long-term survivors. AIDS Res Hum Retroviruses 1999, 15:1219-1228.

22. Propato A, Schiaffella E, Vicenzi E, et al.: Spreading of HIV-specific CD8+ T-cell repertoire in long-term nonprogressors and its role in the control of viral load and disease activity. Hum Immunol 2001, 62:561-576.

23. Kaul R, Rowland-Jones SL, Kimani J, et al.: New insights into HIV-1 specific cytotoxic T-lymphocyte responses in exposed, persistently seronegative Kenyan sex workers. Immunol Lett 2001, 79:3-13.

24. Betts $M$, West $S$, Nason $M$, et al.: The functional hierarchy of the HIV-specific CD8+ T-cell response is dominated by MIP1. Paper presented at the AIDS Vaccine Conference. Lausanne, Switzerland; August 30, 2004.

25. Calarota SA, Weiner DB: Enhancement of human immunodeficiency virus type 1-DNA vaccine potency through incorporation of T-helper 1 molecular adjuvants. Immunol Rev 2004, 199:84-99.

26. Barouch DH, Santra S, Schmitz JE, et al.: Control of viremia and prevention of clinical AIDS in rhesus monkeys by cytokine-augmented DNA vaccination. Science 2000, 290:486-492.

27. Boyer JD, Kutzler M, Robinson T: Improved cellular immune potency by Th1 cytokine adjuvanted DNA vaccines in non-human primates. Paper presented at the AIDS Vaccine Conference. New York, NY; September 18, 2003.

28. Seth A, Ourmanov I, Schmitz JE, et al.: Immunization with a modified vaccinia virus expressing simian immunodeficiency virus (SIV) Gag-Pol primes for an anamnestic Gag- specific cytotoxic T-lymphocyte response and is associated with reduction of viremia after SIV challenge. $J$ Virol 2000, 74:2502-2509.

29. Shiver JW, Fu TM, Chen L, et al.: Replication-incompetent adenoviral vaccine vector elicits effective anti-immunodeficiency-virus immunity. Nature 2002, 415:331-335.

30. Patterson LJ, Malkevitch N, Venzon D, et al.: Protection against mucosal simian immunodeficiency virus SIV (mac251) challenge by using replicating adenovirus-SIV multigene vaccine priming and subunit boosting. J Virol $2004,78: 2212-2221$. 
31. Johnson PR, Schnepp BC, Connell MJ, et al.: Novel adenoassociated virus vector vaccine restricts replication of simian immunodeficiency virus in macaques. $J$ Virol 2005, 79:955-965.

32. Rose NF, Marx PA, Luckay A, et al.: An effective AIDS vaccine based on live attenuated vesicular stomatitis virus recombinants. Cell 2001, 106:539-549.

33. Egan MA, Chong SY, Rose NF, et al.: Immunogenicity of attenuated vesicular stomatitis virus vectors expressing HIV type 1 Env and SIV Gag proteins: Comparison of intranasal and intramuscular vaccination routes. AIDS Res Hum Retroviruses 2004, 20:989-1004.

34. Mascola JR, Sambor A, Beaudry K, et al.: Neutralizing antibodies elicited by immunization of monkeys with DNA plasmids and recombinant adenoviral vectors expressing human immunodeficiency virus type 1 proteins. J Virol 2005, 79:771-779.

35. Sun Y, Schmitz JE, Acierno PM, et al.: Multiclade human immunodeficiency virus type 1 envelope immunogens elicit broad cellular and humoral immunity in rhesus monkeys. J Virol 2005, 79:2956-2963.

36. Smith JM, Amara RR, McClure HM, et al.: Multiprotein HIV type 1 clade $B$ DNA/MVA vaccine: construction, safety, and immunogenicity in Macaques. AIDS Res Hum Retroviruses 2004, 20:654-665.

37. Mehandru S, Poles MA, Tenner-Racz K, et al.: Primary HIV-1 infection with preferential depletion of CD4+ T lymphocytes from effector sites in the gastrointestinal tract. J Exp Med 2004, 200:761-770.

38. Brenchley JM, Schacker TW, Ruff LA, et al.: CD4+ T cell depletion during all stages of HIV disease occurs predominantly in the gastrointestinal tract. J Exp Med 2004, 200:749-759.

39.• Mattapallil JJ, Douek DC, Hill B, et al.: Massive infection and loss of memory CD4 + T cells in multiple tissues during acute SIV infection. Nature 2005, 434:1093-1097.

This paper shows that SIV rapidly and selectively infects and destroys memory CD4+ T lymphocytes within days of infection. This results in loss of T lymphocytes with a memory phenotype of CD4+ CCR5+. The major focus of this destruction is in mucosal tissues, where most of T lymphocytes expressing CD4 and CCR 5 reside.

40.• Li Q, Duan L, Estes JD, et al.: Peak SIV replication in resting memory $\mathrm{CD} 4+\mathrm{T}$ cells depletes gut lamina propria CD4+ T cells. Nature 2005, 434:1148-1152.

This paper also shows that SIV quickly and specifically infects and destroys memory CD $4+\mathrm{T}$ lymphocytes within the first 4 days postinfection. This event causes memory CD4+ CCR5+ T lymphocyte

loss at mucosal sites where most of these lymphocytes reside.
41. Musey L, Hu Y, Eckert L, et al.: HIV-1 induces cytotoxic T lymphocytes in the cervix of infected women. J Exp Med 1997, 185:293-303.

42. Kaul R, Plummer FA, Kimani J, et al.: HIV-1-specific mucosal CD8 + lymphocyte responses in the cervix of HIV-1-resistant prostitutes in Nairobi. J Immunol 2000, 164:1602-1611.

43. Musey L, Ding Y, Cao J, et al.: Ontogeny and specificities of mucosal and blood human immunodeficiency virus type 1-specific CD8+ cytotoxic T lymphocytes. J Virol 2003, 77:291-300.

44. Bomsel M, Heyman M, Hocini H, et al.: Intracellular neutralization of HIV transcytosis across tight epithelial barriers by anti-HIV envelope protein dIgA or IgM. Immunity 1998, 9:277-287.

45. Picker LJ, Hagen SI, Lum R, et al.: Insufficient production and tissue delivery of CD4+ memory $\mathrm{T}$ cells in rapidly progressive simian immunodeficiency virus infection. J Exp Med 2004, 200:1299-1314.

46. Anton PA, Mitsuyasu RT, Deeks SG, et al.: Multiple measures of HIV burden in blood and tissue are correlated with each other but not with clinical parameters in aviremic subjects. AIDS 2003, 17:53-63.

47. Guadalupe M, Reay E, Sankaran S, et al.: Severe CD4+ T-cell depletion in gut lymphoid tissue during primary human immunodeficiency virus type 1 infection and substantial delay in restoration following highly active antiretroviral therapy. J Virol 2003, 77:11708-11717.

48. George MD, Reay E, Sankaran S, Dandekar S: Early antiretroviral therapy for simian immunodeficiency virus infection leads to mucosal CD4+ T-cell restoration and enhanced gene expression regulating mucosal repair and regeneration. J Virol 2005, 79:2709-2719.

49. Belyakov IM, Hel Z, Kelsall B, et al.: Mucosal AIDS vaccine reduces disease and viral load in gut reservoir and blood after mucosal infection of macaques. Nat Med 2001, 7:1320-1326.

50. Musey L, Ding Y, Elizaga M, et al.: HIV-1 vaccination administered intramuscularly can induce both systemic and mucosal T cell immunity in HIV-1-uninfected individuals. J Immunol 2003, 171:1094-1101. 